\title{
GENERACIÓN DE RECURSOS DIDÁCTICOS Y MULTIMEDIA PARA EL DESARROLLO DE HABILIDADES APTITUDINALES EN ASPIRANTES A LAS INSTITUCIONES DE EDUCACIÓN SUPERIOR EN LA PROVINCIA DE CHIMBORAZO
}

M.Sc. Sánchez Ortiz Silvia Magdalena. ${ }^{1}$ Lic. Anilema Troya Edwin Stalin. ${ }^{2}$ Lic.Psic. Galarza Lozada Ángel Marcelo. ${ }^{3}$ Lic. Cushpa Inchiglema Raúl Clemente. ${ }^{4}$ Lic.Barba Tamayo Edison Paúl ${ }^{5}$

\begin{abstract}
Historically Ecuadorian education has aimed to measure knowledge and content, so the preparation was directed to the immediate storage of information whose mechanism was more efficient memorism. In assuming this new challenge proposed by the State, there is no doubt that it is necessary to change completely the way to prepare for this examination, and although the skills can not be studied as such, if they can be exercised, developed and also if the student is more familiar with these kinds of questions will have better results. Unfortunately, this academic preparation has been marketed with the provision of preparation courses at very expensive costs, so the present project is consolidated as a free didactic contribution that using information and communication technologies helps the aspirant to prepare for the national evaluation. This project generated three didactic resources that serve as learning tools: A) Guide for the aspirant, who from psychology seeks to help the emotional control, delivering several tips. so that the student generates self-confidence and can perform better, as well as transcendental information about the process, this guide can be printed as well as uploaded to the internet to increase its coverage, B) Video tutorial generated in 2D in which SENESCYT exercises are solved, to generate a demonstrative learning, this multimedia resource can be uploaded to communities that share videos in the world wide web and C) tangible didactic material that becoming playful objects allow the development of aptitudinal exercises, that can be used in classes, fairs, lectures and conferences. The present project is conceived as a small initiative that can be promoted under the tutelage and sponsorship of institutions of higher education that can inject resources to this initiative, for example through its lines, programs and projects of linkage.
\end{abstract}

\section{Keywords:}

Resources, Didactics, Multimedia, Development, Aptitudinal Skills, Institutions Of Higher Education

\section{CÓDIGO UNESCO: Educación 531204}

\footnotetext{
${ }^{1}$ Unidad Educativa Picaihua; Ambato, Ecuador; silviasan 16@yahoo.es

${ }^{2}$ Unidad Educativa Pedro Vicente Maldonado; Ambato, Ecuador; dufamapola@yahoo.com

3 Instituto Pedagógico de Salid Integral; Ambato, Ecuador; angelitop6@hotmai.es

${ }^{4}$ Corporación Cruzada Social; Ambato, Ecuador; raulcushpa@gmail.com

${ }^{5}$ Universidad Politécnica de Chimborazo; Ambato, Ecuador; edybarba16@hotmail.com
} 


\section{RESUMEN}

Históricamente la educación ecuatoriana ha apuntado medir conocimientos y contenidos, por ende la preparación se dirigía al almacenamiento inmediato de información cuyo mecanismo más eficiente era el memorismo. Al asumir este nuevo desafío propuesto por el Estado es indudable que es necesario cambiar por completo la manera de prepararse para este examen, y a pesar que las aptitudes no pueden ser estudiadas como tal, si pueden ser ejercitadas, desarrolladas y así como también si el estudiante está más familiarizado con este tipo de preguntas tendrá mejores resultados.

Lastimosamente esta preparación académica se ha visto mercantilizada con la oferta de cursos de preparación a costos muy onerosos, por eso el presente proyecto se consolida como un aporte didáctico gratuito que utilizando las tecnologías de información y comunicación ayuda al aspirante a prepararse para la evaluación nacional. Este proyecto se generó tres recursos didácticos que sirven como herramientas de aprendizaje: A) Guía para el aspirante, que desde la psicología busca ayudar al control emocional, entregando varios tips. para que el discente genere autoconfianza y puedas rendir mejor, así como entrega información trascendental acerca del proceso, esta guía puede ser impresa como también subida al internet para aumentar su cobertura, B) Video tutorial generado en 2D en la cual se resuelven ejercicios tipo SENESCYT, como para generar un aprendizaje demostrativo, este recurso multimedia puede ser subido a comunidades que comparten videos en la world wide web y C) Material didáctico tangible que convirtiéndose en objetos lúdicos permiten el desarrollo de ejercicios aptitudinales, que puede ser usado en clases, ferias, charlas y conferencias. El presente proyecto se concibe como una pequeña iniciativa que puede ser potenciada bajo la tutela y auspicio de instituciones de educación superior que puedan inyectar recursos a esta iniciativa, por ejemplo mediante sus líneas, programas y proyectos de vinculación.

\section{Palabras clave:}

Recursos, Didáctica, Multimedia, Desarrollo, Habilidades Aptitudinales, Instituciones de Educación Superior

\section{INTRODUCCIÓN}

\section{DESCRIPCIÓN DETALLADA DEL PROYECTO}

La Ley Orgánica de Educación Superior (LOES) y su respectivo reglamento en vigencia desde el año 2010 establecieron la creación inédita en la historia de nuestro país del Sistema Nacional de Nivelación y Admisión (SNNA), con el objeto de homogenizar el mecanismo de acceso a las universidades, democratizar el acceso a la educación superior y asignar los cupos ofertados por las instituciones de Educación Superior por mecanismos meritocráticos, este sistema tiene dos componentes básicos: admisión y nivelación, al hablar del primero tiene como características ser permanente, nacional y unificado.

En la actualidad el proceso de admisión a las instituciones de educación superior se encuentra normado por el Sistema Nacional de Nivelación y Admisión (SNNA) quién administra meritocráticamente los cupos ofertados por las instituciones de Educación Superior, conforme a la selección de estudiantes, considerando sus aptitudes y habilidades del pensamiento. 
El instrumento de evaluación para otorgar el cupo es el Examen nacional para la educación superior (ENES), el mismo que está compuesto por 120 reactivos en tres grandes áreas: razonamiento numérico, abstracto y verbal, buscando evaluar habilidades aptitudinales y no contenidos disciplinares.

Este proceso ocasiona una ruptura con los tradicionales instrumentos evaluativos de admisión, que apuntaban a un desarrollo asignaturista y memorístico en los postulantes. Entre los desafíos del nuevo sistema de admisión es generar confianza y adaptabilidad a las nuevas formas evaluativas, ya que los estudiantes no han estado acostumbrados a este tipo de exámenes. Esto conlleva a la búsqueda de apoyo para mejorar las aptitudes con miras a rendir el Examen Nacional de Educación Superior.

Los profundos cambios sociales, tecnológicos han permitido el desarrollo de propuestas metodológicas basadas para ayudar a la comunidad estudiantil en sus procesos de aprendizaje y actualización. El reto está entonces en hacer uso adecuado de los recursos didácticos multimedia para potenciar habilidades, afianzar conocimientos, mejorar las estrategias, a través de herramientas que se encuentren al alcance de todos los estratos sociales, sin restricción alguna.

De acuerdo a un estudio realizado por el Instituto Nacional de Estadística y Censos, INEC, (2012): “(...) el Ecuador cuenta con 555.782 jóvenes universitarios; 346.326 estudian en instituciones públicas, 62.018 en universidades privadas y 147.438 en cofinanciadas. La gratuidad y el mejoramiento de la calidad académica son los principales motivos por los que la gran mayoría de los ecuatorianos elige universidades públicas"(s/p)

Para el mismo INEC (2012): “(...) El Examen Nacional para la Educación Superior permitió que en 2012 la tasa de ingreso y matriculación a la educación superior suba al 71\%. En 2009 y 2010 la cifra de ingreso de aspirantes fue del 54\% y 55\% respectivamente"(s/p)

Este nuevo sistema permitió aumentar la tasa de matrícula a la Universidad de grupos históricamente excluidos, esto ha generado que el Ecuador sea un pionero en la inclusión educativa en las instituciones de educación superior.

Es imprescindible mencionar, que a pesar que la tasa de matrícula se ha incrementado tres universidades: Universidad Estatal de Guayaquil, Universidad Técnica de Manabí y Universidad Técnica de Ambato reportaron en el año 2011 un descenso de alumnos de hasta el treinta por ciento.

Las cifras de tasa neta de matriculación en educación superiorl aún no puede ser comparada en rengos históricos, ya que los instrumentos estadísticos del INEC, contemplaban interrogantes correspondientes a otros sistemas educativos que fueron derogados, por ende el verdadero resultado de los nuevos proceso de admisión están en fase de implementación y ejecución, evaluable de forma mediata recién en los próximos años.

El presente proyecto busca ser una alternativa de ayuda gratuita y de fácil acceso para coadyuvar a desarrollar habilidades aptitudinales en los estudiantes a través de recursos didácticos y multimedia, para luchar contra el negocio fructífero y lucrativo que se ha generado al preparar a estudiantes para ser admitidos en educación superior. 
La tasa neta es un indicador que mide la cantidad de estudiantes matriculados en establecimientos de nivel superior y que pertenecen al grupo de edad que, según las normas educativas, corresponde a ese nivel (de 18 a 24 años). Va en función de la población.

Esta iniciativa tiene tres componentes didácticos que utilizando las tecnologías de la información y comunicación, busca consolidarse en un plataforma de aprendizaje y preparación de aptitudes y razonamientos, el primer componente nace desde la necesidad psicológica y emocional para enfrentar una evaluación tan determinante en la vida de una persona como es la de obtener un cupo en la educación superior en la carrera de preferencia, dicho componente, brinda sugerencias que permitan a los aspirantes comprender los temas abordados por el SNNA, además señala ciertas pautas para que los bachilleres puedan realizar el examen de ingreso a las instituciones de nivel superior. El segundo componente nace desde el aspecto lúdico, que sirve como punto de partida para entender el razonamiento verbal y abstracto desde materiales tangibles que permitan al aspirante desarrollar su capacidad de razonamiento para comprender los procesos aplicados en los reactivos utilizados en el examen. Así, los conocimientos adquiridos en el bachillerato serán razonados adecuadamente en la resolución de los problemas planteados, es preciso señalar que este sistema de admisión tiene como finalidad otorgar las mismas oportunidades a todos los bachilleres provenientes de todas las unidades educativas del país y finalmente el video-tutorial que es la herramienta multimedia de mayor rendimiento y menor costo, el cual, permite masificar algunas alternativas de solución en las preguntas planteadas en el examen, es necesario resaltar que las herramientas multimedia en la actualidad son indispensables para los procesos de aprendizaje y son de fácil acceso y manejo para la mayoría de bachilleres.

Este proyecto busca sensibilizar y concientizar sobre la necesidad de alcanzar el puntaje necesario para ubicarse en la carrera escogida por los postulantes, brindando herramientas factibles, aplicando ideas sencillas y prácticas, de fácil alcance y comprensión para la resolución de los reactivos aplicados en los tres tipos de razonamientos.

Es necesario resaltar la importancia de una educación superior en los tiempos actuales, el desarrollo de competencias que permitan al bachiller contribuir a la solución de una gran problemática sobre todo de índole económica ya que muchos de los estudiantes no cuentan con los recursos necesarios para escoger una capacitación particular por los elevados costos que tienen, la obligatoriedad va de la mano con la gratuidad; por tanto, este proyecto surge como una alternativa de solución ante la problemática que se genera para la preparación que los estudiantes requieren para el Examen Nacional de Educación Superior.

Se plante los siguientes objetivos generales y específicos del proyecto: Recursos didácticos y multimedia mediante herramientas físicas y de software, que sirvan para el desarrollo de habilidades aptitudinales en los aspirantes a las Instituciones de Educación Superior.

- Aplicar estrategias didácticas mediante el uso de la tecnología para facilitar la comprensión verbal, lógico matemático y abstracto.

- Desarrollar recursos didácticos multimedia a través de aplicaciones informáticas que permitan un aprendizaje lúdico del razonamiento verbal, lógico y abstracto. Emplear las 
aplicaciones multimedia en talleres que coadyuven a la formación de los aspirantes a las instituciones de educación superior, mediante un sistema abierto, virtual y a distancia.

\section{FUNDAMENTACIÓN CIENTÍFICA TÉCNICA}

\section{Generación}

El Diccionario de la Real Academia Española de la Lengua (2014) define al termino de generación de la siguiente manera: "Acción que consiste en producir o crear una cosa. Avances e innovaciones respecto a la fase anterior. " (p.175)De acuerdo al criterio de Robalino, P. (2014) Generar es diseñar, reconocer y crear patrones creativos, formular metas y crear estrategias para lograrla. p.38.

\section{Recursos Didácticos}

El recurso didáctico es un instrumento o medio por el que acontece la comunicación entre catedráticos y educandos. Son recursos instrumentales que inciden en la transferencia educativa, y tienen sólo sentido cuando se piensan en relación con el aprendizaje. (Jaume, 2012).

Los recursos didácticos son distintos elementos que pueden agruparse en un conjunto, reunidos de acuerdo a su utilización en algún fin específico. Estos pueden ser reales (físicos), virtuales o abstractos. (Auquilla, T. 2012).

"Los recursos didácticos constituyen un recurso útil para favorecer procesos de aprendizaje de habilidades, de actitudes, de conocimientos,... siempre que se conciban como un medio al servicio de un proyecto que se pretende desarrollar" (Parcerisa, A. 2010).

Los recursos didácticos se caracterizan por despertar el interés del estudiante adaptándose a sus características, para facilitar la labor docente $\mathrm{y}$, por ser sencillo, consistente y adecuado a los contenidos, son herramientas que nos ayudan al proceso enseñanza- aprendizaje.

Para el uso de las herramientas didácticas se debe tomar en cuenta muchos factores que influirán en la selección y posterior utilización de estos medios, factores, muchos de ellos ajenos a los propios medios, como son la disponibilidad de estos y otros que dependen tanto de los alumnos como del lugar donde vamos a impartir la docencia y, en este último caso, de las condiciones ambientales en las que la clase se desarrolla. Aspectos tales como la situación de los asientos con respecto a la pantalla o pantallas, la posibilidad de oscurecer el aula, las condiciones acústicas, etc. Pueden obligar a elegir unos recursos frente a otros e, incluso, prescindir de éstos.

\section{Clasificación de los Recursos Didácticos}

Siguiendo la clasificación de Moreira, M. (2003) podemos encontrar las siguientes clases de recursos didácticos:

Escritos.- Establecido en la ubicación sucesiva de caracteres que acatan a un código lingüístico. Lo escrito persiste y si se lo expresa de una manera directa tiene una gran incidencia. En la actualidad existen muchos mecanismos de transmisión de información, pero sigue siendo el texto el mejor mecanismo para el aprendizaje. 
Visual.- La imagen surge ante las personas y causa un impacto total, ya que no necesita un proceso secuencial como es la escritura. Sin embargo, los significados de una imagen se condicionan por diversas circunstancias internos y externos.

Escrito-visual.- Este sistema combina y complementa la imagen junto al texto. La ocupación del texto es añadir una nueva dimensión de valor icónica.

Sonoro o auditivo.- El lenguaje oral es la mejor manera de comunicación humana, es la herramienta básica en un proceso de aprendizaje.

Audiovisuales: hacen uso de la imagen y el sonido para la presentación de contenidos.

Multimedia: Los autores Alonso, M. \& Padilla L. (2005) define "que utiliza conjunta y simultáneamente diversos medios como imágenes, sonidos y texto, en la transmisión de una información", teniendo en cuenta los siguientes aspectos técnicos:

a) Calidad del entorno audiovisual.

b) Calidad y cantidad de los elementos multimedia.

c) Calidad y estructura de los contenidos.

d) Estructura y navegación por las actividades.

e) Interacción.

f) Ejecución fiable y visualización adecuada.

Bravo, J. (2004) Los sistemas multimedia, son una nueva concepción del aprendizaje donde el alumno construye los contenidos creando sus propias significaciones en un diálogo continuo con el sistema. Además, estos medios de extraordinaria flexibilidad, permiten la evaluación continua del proceso y la evaluación final, debiendo tomar en cuenta los siguientes aspectos:

Interactividad, el usuario interactúa sobre el medio de manera que éste sea un sujeto activo y no un mero receptor de mensajes.

Iconicidad.- se refiere al objeto representado tal y como es.

Sincronía o a sincronía. Un medio sincrónico es aquel que permite la comunicación entre el emisor y receptor cuando ambos están conectados simultáneamente, permitiendo el intercambio continuo de papeles.

También se habla de los Tutores multimedia. Empleando todas las posibilidades de comunicación de las tecnologías multimedia y pueden contener grandes volúmenes de información que llegan al usuario a través de un soporte sencillo, barato, extendido, fácil de manejar y de gran capacidad como es el CD-ROM. (Bravo, J. 2004)

El vídeo educativo, ofrece una información distribuida mediante un soporte físico y de gran capacidad de almacenamiento. Es un soporte barato, robusto, fácil de emplear y que no necesita instalación. Sus niveles de interacción son muy bajos y, por ello, es un soporte muy adecuado para transmitir una información lineal que debe ser captada por el alumno en un determinado orden. (Bravo, J. 2004) 


\section{Selección de Materiales Educativos}

En la actualidad disponemos de una gran variedad de recursos siendo indispensable distinguir que tipo de recurso es el más apropiado para cada momento, conociendo sus ventajas y desventajas y en qué medida se ajusta a las características del contenido que va abordar, de los alumnos que va dirigida la actividad y de sus propias características y capacidades como docente.

Para tomar la decisión sobre qué tipo de material utilizar o elaborar, Mediano, F. (2010) comparte los siguientes parámetros:

- Objetivos que pretende conseguir.

- Contenidos que se va abordar.

- Características del alumnado.

- El contexto en el que nos encontramos.

- Las estrategias que vamos a utilizar.

\section{Habilidades Aptitudinales}

Cabe destacar que las aptitudes de una persona son como una cajita de herramientas a las cuales la persona siempre recurre para solucionar lo que le toque enfrentar y son útiles para una variedad de tareas o actividades. Además no se trata que una persona tenga o no tenga una determinada aptitud, todos la tenemos pero en diferentes grados.

\section{Pensamiento abstracto}

La medida de las habilidades cognitivas ha sido estudiada por diferentes autores desde principios del siglo pasado. Así, Binet (como se cita en Bennett, \& Wesman, 1997).

Según Colom \& García López (2002) se manifiesta que "Actualmente, hay cierto consenso sobre el hecho de que dichas diferencias se pueden encontrar en ciertas habilidades, particularmente las espaciales, pero no en la inteligencia general.” Según la guía del SNNA (1012) el razonamiento abstracto involucra la capacidad o aptitud para resolver problemas lógicos deduciendo ciertas consecuencias de una situación.

Es como si la idea de la globalización hubiese llegado a este campo de estudio de la Psicología, donde la inteligencia está muy vinculada con la emoción, la memoria, la creatividad, el optimismo, la personalidad y en cierto sentido con la salud mental las emociones. La inteligencia artificial investiga cómo los ordenadores pueden procesar los aspectos emocionales de una narración.

Por consiguiente y de cualquier modo este debate ha servido también para mejorar la definición de inteligencia y los métodos de análisis, consiguiéndose además una relativa confluencia de posiciones en torno de la diferenciación inteligencia fluida (Gf)- inteligencia cristalizada (Gc) y de los modelos teóricos que de aquí han surgido, por ejemplo, la teoría de los tres estratos o teoría Cattell-Horn-Carroll (Carroll, 1993; McGrew y Flanagan, 1998). Básicamente se propone que la inteligencia, en términos de su estructura, debe ser comprendida por un modelo de tres estratos, 
organizados jerárquicamente, situándose en un nivel superior o más general el propio factor "g", un nivel intermedio formado por una decena de factores de segunda orden asociados a procesos cognitivos específicos (velocidad de procesamiento, capacidad de almacenamiento, evocación de información, raciocinio...) o a contenidos de las tareas (numérico, viso-espacial, auditivoverbal...), y finalmente un conjunto bastante más numeroso y heterogéneo de factores primarios o de primera orden, más próximos de las especificidades de los tests y tareas usadas en la evaluación cognitiva (Carroll, 1997).

\section{METODOLOGÍA}

El proyecto contiene un conjunto de actividades interrelacionadas, coordinadas y dirigidas a lograr como resultado mejoras en el desarrollo de habilidades aptitudinales en los aspirantes a las Instituciones de Educación Superior a corto plazo y con recursos propios. Además se aplica el método inductivo-deductivo de tal manera que el análisis de requerimientos se realiza de lo general a lo particular con ello se trata de aportar con herramientas concretas en la búsqueda de soluciones al problema de la mercantilización de cursos y talleres para el ingreso a la educación superior.

El proyecto tiene como objetivo ayudar a la capacitación en ambientes flexibles, entornos virtuales y sin costo alguno. La elaboración de materiales y recursos didácticos para su implementación a través de los medios tecnológicos como internet permitirá capacitar a los interesados en ejercicios similares a los propuestos en el Examen Nacional para la Educación Superior.

La metodología utilizada también es un conjunto de técnicas para garantizar el desarrollo del producto multimedia se lleve a cabo de forma organizada y eficiente, determinando en gran medida el éxito de los resultados esperados para la capacitación de aspirantes a las IES públicas la misma que se describe a continuación:

\section{ANÁLISIS PRELIMINAR}

En esta primera etapa se realizó conversatorios con algunos estudiantes y docentes del SNNA de información básica preliminar relacionada con la idea del proyecto "Elaboración de recursos didácticos y multimedia. Esta información fue analizada por nuestro equipo de trabajo con la finalidad de decidir la factibilidad y pertinencia de la propuesta para luego continuar con las siguientes etapas de la elaboración del proyecto.

\section{DIAGNÓSTICO}

Se elaboró un pequeño esquema para determinar la importancia, tema, objetivos teniendo como principios fundamentales para esta etapa el impacto social. Para la propuesta se analizó de manera preliminar los siguientes aspectos: Prioridades locales, Viabilidad Técnica y Económica.

APROBACIÓN DEL DIAGNÓSTICO 
El conjunto de ideas y sugerencias sobre problemas en el ámbito educativo fueron analizadas y discutidas por los integrantes del grupo de trabajo con la finalidad de aprobar y continuar con las siguientes etapas de la elaboración del proyecto.

\section{BENEFICIARIOS Y GRUPOS DE INTERÉS}

Se consideran beneficiaros a los estudiantes de tercer año de bachillerato, docentes del SNNA y bachilleres aspirantes a las Instituciones de Educación Superior.

\section{ETAPAS DE LA METODOLOGÍA PARA EL DESARROLLO DE PROYECTOS MULTIMEDIA}

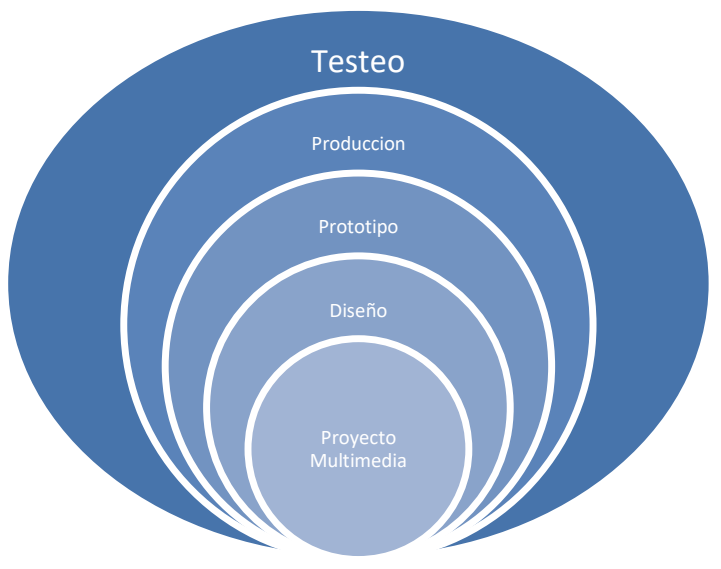

Grafico N¹: Etapas De La Metodología Para El Desarrollo De Proyectos Multimedia

Elaboración.- Grupo de investigación

\section{ACTIVIDAD TÉCNICA U OPERACIONAL PARA DESARROLLO DEL} PROYECTO.

En esta etapa se describe cómo se elaboró los recursos didácticos y multimedia:

\section{ETAPA IDEA}

En esta etapa se determinaron los objetivos del proyecto, perfil de usuario y plataformas tecnológicas para el diseño de recursos didácticos y multimedia dirigido a estudiantes y bachilleres.

a. Determinar el contenido a tratar (Verbal, Numérica y Abstracta)

b. Se analizó estrategias para facilitar la comprensión verbal, lógico matemático y abstracto.

c. Revisión de los contenidos del ENES y selección de los ejercicios de aptitud verbal, numérica y abstracta.

d. Se definió las estrategias tecnológicas para elaborar ejercicios y el tiempo de duración del material multimedia. 
e. Se procedió a seleccionar los ejercicios tipo, uno por cada categoría.

\section{ETAPA DISEÑO}

En esta etapa se definieron principalmente los ejercicios relacionados con aptitud verbal, numérica y abstracta; de igual manera se elaboró un guion técnico interactivo para realizar las animaciones multimedia.

a. Se identificaron el tipo de aplicaciones existentes en el medio y como aportan a la preparación y autoaprendizaje de los aspirantes.

b. Revisión de los materiales existentes en internet y su efectividad en el aprendizaje.

c. Se revisaron sitios web y recursos digitales que proporcional el SNNA.

d. Selección de las herramientas tecnológicas para elaborar el video tutorial.

e. Instalar y configurar las aplicaciones de soporte informático.

f. Diseñar una aplicación multimedia que proporcione facilidad y rapidez a la solución de problemas planteados en las evaluaciones de ingreso a las universidades.

g. Realizar un análisis de requerimientos: propagandas, blogs, trípticos.

h. Seleccionar la metodología de desarrollo multimedia y su guion.

i. Programación y animación digital.

\section{ETAPA PROTOTIPO}

Se genera un prototipo de la aplicación multimedia o video con el objetivo de garantizar a los estudiantes y bachilleres que los recursos didácticos sean amigables y de fácil acceso; desarrollados dentro de los objetivos planteados en el proyecto para posteriormente probar la aplicación a través de sitios web a fin de que sea utilizado por los aspirantes.

\section{ETAPA PRODUCCIÓN}

Esta etapa del proyecto se elaboró las imágenes, animaciones, videos del recurso didáctico multimedia. Se definieron los criterios de producción y se realizaron los diversos elementos multimediales de acuerdo al guion técnico interactivo y con el apoyo de herramientas informáticas para multimedia educativa.

Se diseñó la guía metodológica la misma que contiene:
a. Introducción
b. ¿Estar bien informado?
c. ¿Definiendo estados emocionales?
d. Tener una técnica eficaz
e. Realizar una preparación mental

\section{ETAPA TESTEO O PRUEBAS}

Se realizaron pruebas con el objetivo de verificar el adecuado funcionamiento y la calidad del video con respecto al contexto en donde operará. Esto incluye el proceso de encontrar errores en el desarrollo de animaciones además este proceso se relaciona a atributos como la fiabilidad, eficiencia, usabilidad y capacidad del mismo. Evaluación y versión definitiva. 


\section{ETAPA 5 DISTRIBUCIÓN}

¿La distribución de los recursos didácticos y multimedia se realiza por diversos medios, tales como Internet, redes sociales, blogs cd-roms o sitios interactivos de consulta a través de las tecnologías de comunicación en la World Wide Web?
a. Entregar trípticos con detalles del proyecto.
b. Publicar en la web los recursos didácticos multimedia.
c. Elaborar el proyecto en su totalidad.
d. Revisión y publicación en la revista de proyectos UTA
e. Presentación en la Feria de Proyectos "Yawpakman Rikuna" CEPOS-UTA
f. Publicación final en los medios como internet.
g. Grabación de CD multimedia y guía de orientación psicológica.

\section{FACTIBILIDAD}

La necesidad de proporcionar materiales educativos concretos con la finalidad de ayudar a los aspirantes en el proceso de formación y preparación previa a su ingreso a las instituciones de educación superior, además para el desarrollo del proyecto educativo se cuenta con los recursos materiales, tecnológicos y económicos que permiten su desarrollo e implementación de manera exitosa.

\section{EJECUCIÓN, SUPERVISIÓN Y SEGUIMIENTO}

El proyecto se elabora utilizando herramientas informáticas multimedia para posteriormente publicarlas a través de sitios gratuitos de la web 2.0. Para complementar la fase de presentación la dirección de Posgrado de la UTA realizará una Feria de proyectos en la cual se dará a conocer a comunidad universitaria y ciudadanía en general esta propuesta de innovación y desarrollo.

\section{EVALUACIÓN Y CONTROL}

Se realiza evaluación de la efectividad de los recursos didácticos y multimedia diseñados de manera intuitiva para facilitar el aprendizaje y desarrollo aptitudinal en los aspirantes a las IES éste proceso tendrá resultados mediante la publicación de recursos en internet donde los usuarios podrán comentar y sugerir sobre la efectividad de los recursos elaborados.

\section{RESULTADOS ESPERADOS}

Que el 70\% de los docentes de la ciudad de Ambato conozcan sobre estos recursos didácticos y multimedia a los maestros de tercer año de bachillerato para incentivar a los estudiantes en el uso adecuado de la tecnología.

Que el $80 \%$ de los estudiantes estén conscientes de que existen estrategias metodológicas en materiales multimedia para la comprensión verbal, lógico matemático y abstracto.

Los principales productos de la ejecución de esta propuesta se resumen de la siguiente manera:

- Una guía de orientación psicológica para los aspirantes a las instituciones de educación superior. 
- Recursos didácticos multimedia como herramientas para el autoaprendizaje dirigido a docentes y estudiantes de bachillerato.

- Aplicación multimedia que proporcione facilidad y rapidez a la solución de

- problemas planteados en las evaluaciones de ingreso a las universidades tal como

- propone el SNNA.

- Participación en la Feria de Proyectos Educativos organizado por el Centro de

- Posgrado de la Universidad Técnica de Ambato para posteriormente publicarlo en una revista elaborada por el CEPOS -UTA.

- Material gratuito para la preparación de aspirantes a las instituciones de educación

- superior a través de revista electrónica en internet, afiches y trípticos.

- CD multimedia con la recopilación de los recursos didácticos elaborados en la

- propuesta.

\section{FINANCIAMIENTO}

\begin{tabular}{|c|c|c|c|c|c|c|c|}
\hline $\begin{array}{l}\text { Tipo } \\
\text { Compra }\end{array}$ & Detalle del Producto & $\begin{array}{c}\text { Cantidad } \\
\text { Anual }\end{array}$ & Unidad & $\begin{array}{l}\text { Costo } \\
\text { Unitario }\end{array}$ & $\begin{array}{l}\text { Cuatrime } \\
\text { stre }\end{array}$ & $\begin{array}{l}\text { Cuatrime } \\
\text { stre }\end{array}$ & $\begin{array}{l}\text { Cuatrime } \\
\text { stre }\end{array}$ \\
\hline BIEN & STORYBOARD & 5 & 1 & 10,00 & & $\mathrm{~S}$ & \\
\hline BIEN & $\begin{array}{c}\text { ANIMACIÓN DE } \\
\text { CADA } \\
\text { EJERCICIO }\end{array}$ & 5 & 1 & 20,00 & $\mathrm{~S}$ & & \\
\hline BIEN & REVISTA & 10 & 5 & 5,00 & & & $\mathrm{~S}$ \\
\hline
\end{tabular}

Tabla $N^{\circ} 1$ : Recurso financieros

Elaboración: Grupo Investigador

\section{PRODUCCIÓN}

\begin{tabular}{|c|c|c|c|c|c|}
\hline Partida & \multirow{3}{*}{$\begin{array}{l}\text { DETALLE DEL } \\
\text { PRODUCTO }\end{array}$} & CANTIDAD & COSTO & SUB & \\
\hline \multirow{2}{*}{$\begin{array}{l}\text { Presupuest } \\
\text { aria }\end{array}$} & & ANUAL & UNITARIO & TOTAL & Valor \\
\hline & & (2a) & (2b) & $(2 a) *(2 b)$ & Solicitado \\
\hline 1 & STORYBOARD & 5 & 10 & 50 & - \\
\hline 2 & $\begin{array}{l}\text { ANIMACIÓN DE CADA } \\
\text { EJERCICIO }\end{array}$ & 5 & 20 & 100 & - \\
\hline 3 & MODELADO 3D & 6 & 30 & 180 & - \\
\hline 4 & DISEÑO & 1 & 300 & 300 & - \\
\hline 5 & IMPRESIÓN & 20 & 10 & 200 & - \\
\hline 6 & REVISTA & 5 & 10 & 50 & \\
\hline \multicolumn{4}{|c|}{ TOTAL RECURSOS FÍSICOS } & 300 & - \\
\hline & & & TOTAL USD. & $1.180,00$ & \\
\hline
\end{tabular}




\section{Tabla $N^{\circ} 1$ : Recurso financieros}

Elaboración: Grupo Investigador

\section{ACTIVIDADES DEL PROYECTO Y TIEMPOS DE EJECUCIÓN}

El proyecto está diseñado para realizarse en un lapso no mayor a los tres mese de acuerdo al siguiente detalle de actividades.

- Analizar estrategias utilizadas para facilitar la comprensión verbal, lógico matemático y abstracto.

- Describir las forma de apoyo virtual que brinda el SNNA a través de su sitio web.

- Identificar las herramientas tecnológicas que se ha utilizado para desarrollar material multimedia.

- Seleccionar la información desde sitios oficiales como SNNA y SENESCYT.

- Revisar los contenidos aptitudinales del ENES

- Clasificar en categorías verbal, numérica y abstracta.

- Seleccionar los ejercicios de aptitud verbal, numérica y abstracta desde el sitio web oficial.

- Definir las estrategias tecnológicas para elaborar ejercicios y animaciones multimedia.

- Realizar el guion técnico para facilitar la diagramación.

- Identificar el tipo de aplicaciones y materiales existentes en el medio y como aportan en el desarrollo de software educativo y recursos didácticos.

- Revisar los materiales existentes en internet y su efectividad en el aprendizaje de los aspirantes a las IES.

- Diseñar una encuesta online para conocer el impacto de la propuesta.

- Seleccionar las herramientas tecnológicas para elaborar el video tutorial y la guía didáctica.

- Instalar y configurar las aplicaciones de soporte informático.

- Diseñar una aplicación multimedia que proporcione facilidad y rapidez a la solución de problemas planteados en las evaluaciones de ingreso a las universidades.

- Realizar un análisis de requerimientos

- Seleccionar la metodología de desarrollo multimedia.

- Elaborar la guía multimedia.

- Imprimir trípticos para promocionar el proyecto.

- Publicar en la web los recursos didácticos multimedia

- Crear una revista digital de acceso libre a través de plataformas de almacenamiento gratuito.

- Creación de un canal en youtube y sitios similares.

- Diseño e incorporación de materiales didácticos multimedia en un blog http://utaeduser.blogspot.com/

Promocionar en las redes sociales el material multimedia.

- Establecer talleres de capacitación gratuita en la IES para motivar a los aspirantes.

- Entregar en CD multimedia los productos generados para su fácil distribución.

- Elaborar el proyecto en su totalidad

- Revisión y publicación en la revista de proyectos UTA

- Presentación en la Feria de Proyectos CEPOS-UTA

- Publicación final en los medios como internet 


\section{COSTOS}

\begin{tabular}{|l|l|}
\hline DETALLE & VALOR USD. \\
\hline Útiles de escritorio & 50,00 \\
\hline Recurso Tecnológico & 500,00 \\
\hline Imprevistos & 150,00 \\
\hline Publicación en medios digitales & 70,00 \\
\hline TOTAL & $\mathbf{7 7 0 , 0 0}$ \\
\hline
\end{tabular}

Tabla $N^{\circ} 2$ : Recurso Materiales

Elaboración: Grupo Investigador

COSTO TOTAL DEL PROYECTO A+B: 1.950,00 dólares americanos.

\section{SOSTENIBILIDAD}

La gerencia del Sistema Nacional de Nivelación y Admisión ha mantenido diálogos con las Instituciones de Educación Superior públicas, incentivando a que sean las Universidades y las Escuelas Politécnicas quienes contribuyan mediante proyectos de vinculación a los bachilleres para su preparación en torno a rendir el examen nacional para la educación superior.

Ante lo expuesto es claramente visible que el presente proyecto no solo que puede ser sostenido en el tiempo, sino que a su vez puede ser nutrido, fortalecido y potenciado, ya que la presente generación de recursos didácticos y multimedia es una demostración que desde la sencillez de crear pequeñas herramientas, al poner de nuestro lado los beneficios de la tecnologías y el internet se puede tener mayor alcance y mayor cobertura sin mayor esfuerzo y con menos recursos.

El utilizar las redes sociales y comunidades en línea como por ejemplo YouTube permite tener un mayor impacto y sostenerse en el tiempo, ejemplificando podemos decir que dar un taller o una clase puede ayudar en el mejor de los casos a unos 50 estudiantes, pero si la misma clase la realizamos en un video tutorial y los subimos a YouTube y lo compartimos mediante las redes sociales, su alcance es ilimitado y el video puede permanecer durante mucho tiempo, es decir se puede reproducir y viralizar sin generación de mayor costo, caso contrario que lo que significaría repetir una clase.

El Consejo de Evaluación, Acreditación y Aseguramiento de la Calidad de la Educación

Superior ha incluido que se otorgara un punto en la evaluación institucional a las Universidades que cumplan con programas de vinculación en torno a la preparación académica de los bachilleres, por ende es factible que el presente proyecto encuentre cabida en una institución y sea cobijado dentro de este marco y puede encontrar mayor fuentes de recursos para perfeccionar y mejorar las 
herramientas, hasta convertirse en un plataforma que asesore gratuitamente a los aspirantes en un sistema abierto, a distancia y on line.

\section{BENEFICIARIOS DEL PROYECTO}

\section{BENEFICIARIOS DIRECTOS}

Serán beneficiarios directos los bachilleres de la ciudad de Ambato que están próximos a rendir el examen ENES de razonamiento matemático, verbal y abstracto, para aspirar a un cupo en una universidad pública del País, en especial los estudiantes de bajos recursos económicos, ya que el proyecto tiene un carácter gratuito.

Serán Beneficiarias las Unidades educativas que cuenten con Bachillerato ya que el material didáctico servirá como apoyo para el desarrollo de dichas capacidades.

Los Docentes que brinden apoyo en el desarrollo del razonamiento matemático, verbal y abstracto a los alumnos de las Unidades Educativas Públicas y Privadas.

\section{BENEFICIARIOS INDIRECTOS}

Serán beneficiarios indirectos los Padres y de familia de los futuros bachilleres los alumnos de bachillerato que se graduarán en los próximos años, en general el sistema de Educación superior del Ecuador.

\section{FACILIDADES DE TRABAJO}

La Dirección de Posgrado de la Universidad Técnica de Ambato consciente de la importancia de la implementación de proyectos que brinde alternativas de solución a los problemas de la sociedad y con la obligación de preparar a hombres y mujeres autónomos y creativos, críticos e innovadores comprometidos en el mejoramiento de las condiciones de vida de la sociedad garantiza una ejecución programática de las actividades a realizarse en el presente proyecto.

La Dirección de Posgrado se encargará de solicitar el espacio adecuado para realizar la presentación de este proyecto, que según lo planificado será el Coliseo de la Institución ubicado en el Campus Ingahurco de esta ciudad de Ambato, sitio en el cual se desarrollará el Primer Encuentro Académico denominado "Yawpakman Rikuna".

Esta Dirección se encargará de realizar una promoción por los medios de difusión con los que cuenta la Universidad Técnica de Ambato, para la difusión del mencionado evento con la ciudadanía, la misma que se llevará a cabo mediante campañas en redes sociales para que este evento tenga la difusión necesario con los beneficiarios directos e indirectos de dicho proyecto.

Se contara con una aprobación y la certificación de las memorias de dicho evento para

lo cual la Dirección de Posgrado realizará los trámites necesarios para avalar dicha Publicación con el reconocimiento de los derechos de autor, para que dicho documento cuente con el respaldo académico necesario.

El proyecto será Financiado por los autores del mismo hasta la presentación en el Primer Encuentro Académico, la implementación del proyecto en otros espacios contará con el apoyo de la Dirección de Posgrado. 


\section{CONCLUSIONES}

El proyecto está centrado en el ser humano, se busca garantizar su derecho a una educación superior, para conseguir un acercamiento al principio rector de nuestro País llamado Sumak Kausay también conocido como Buen Vivir.

En una Sociedad con una visión de igualdad social donde el ser Humano está por encima del capital es necesario que cualquier tipo de derecho sea restringido por el aspecto económico y sobre todo el derecho a una educación de calidad y calidez consagrada en la Carta Magna.

El sistema de Educación Superior ha integrado el Sistema Nacional de Nivelación y Admisión en las Universidades Públicas del País, dicho sistema acoge a todos los Bachilleres que han rendido el Examen Enes dicho examen contempla tres tipos de razonamientos, matemático, verbal y abstracto, competencias que muchos en algunos casos son adquiridas mediante el pago de cursos extra curriculares con altos costos.

Este proyecto tiene un alto grado de responsabilidad social por lo cual brinda un acercamiento al desarrollo de dichas capacidades para que los alumnos postulantes a las universidades cuenten con los elementos necesarios para desarrollar los razonamientos tanto matemático como verbal y abstracto de una manera gratuita contribuyendo así con nuestra sociedad y sobretodo brinda estas herramientas que permitan a los bachilleres del País ingresar a una Institución de nivel superior y así lograr el objetivo del Buen Vivir.

El Estado como garantista de los derechos debe brindar las facilidades para que éstos sean materializados en todos los aspectos de la vida del ser humano, en educación este proyecto surge como una alternativa para democratizar y garantizar el acceso permanente de los bachilleres de la República sobre todo de los segmentos históricamente marginados principalmente por situaciones económicas.

Estas pruebas deben ser aplicadas, explicadas y comprendidas de tal manera, que lejos de causar frustración en el estudiante que no llegara a aprobarlas, lo incentive a una preparación continua, que en lo posterior le permita superarla.

RECEIVED: SEPTEMBER, 2016

REVISED: DECEMBER, 2016

\section{BIBLIOGRAFÍA}

Alonso, M. \& Padilla L. (2005) Aplicaciones de la tecnología de la información y la Comunicación. Aulas de Verano. Egraf S.A p. 83 recuperado https://books.google.es/books?isbn=8436941551

Auquilla, T. (2012). Tesis. Elaboración de material didáctico y su cartilla....Universidad Politécnica Salesiana. Carrera de Pedagogía. Repositorio Digital.

Bravo, J. (2004) Revista de medios y educación, Medios de aprendizaje. 24(II). pp. 113- 
124. Revista Pixel.

Consejo de Evaluación, Acreditación y Aseguramiento de la Calidad en la Educación Superior. (2014). SENESCYT publica Resultados de exámenes ENES. Quito: CEAACES. Recuperado de www.ceaaces.gob.ec/.../la-senescyt-publica-resultados-de-los-examenes-

Instituto Nacional de Estadística y Censos. (2012) Indicadores Sociales. Recuperado de http//.www.inec.gob.ec

Jaume, M. (2012) Cómo analizar los materiales. Cuadernos de Pedagogía. № 203. pp. 88-203

Mediano, F. (2010) Didáctica de la Tecnología. 4. Selección y Elaboración de Material

Educativo. Grao 14. Vol. II

Moreira,M. (2003). De los Webs educativos al Material Didáctico Web. Revista Comunicación y Pedagogía. $\mathrm{N}^{\mathrm{o}}$ 188. Pp.32-38.

Parcerisa, A. (2010). Materiales y recursos didácticos en contextos comunitarios. 1

Edición. Gráo. Barcelona. recuperado https://books.google.es/books?isbn=847827930X p.15

Real Academia Española de la Lengua (2014) (23ª́) Diccionario de la Lengua Española. Recuperado de http://www.rae.es

Robalino, P. (2014) Tesis. Diseño de recursos didácticos .UTA. Facultas Diseño Gráfíco Repositorio Bibliotecas del Ecuador.

Vera, D. (2012) (4ªEd.) Análisis Coyuntural. Revista de medios y educación, pp.113-124

ACCEDER AL SITIO DE PUBLICACIÓN DE LOS RECURSOS DIDÁCTICOS Y MULTIMEDIA http://utaeduser.blogspot.com/

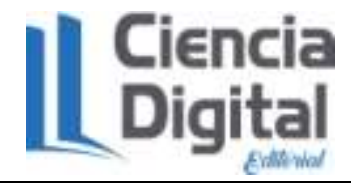

El artículo que se publica es de exclusiva responsabilidad de los autores y no necesariamente reflejan el pensamiento de la Revista Ciencia Digital.

El articulo queda en propiedad de la revista y, por tanto, su publicación parcial y/o total en otro medio tiene que ser autorizado por el director de la Revista Ciencia
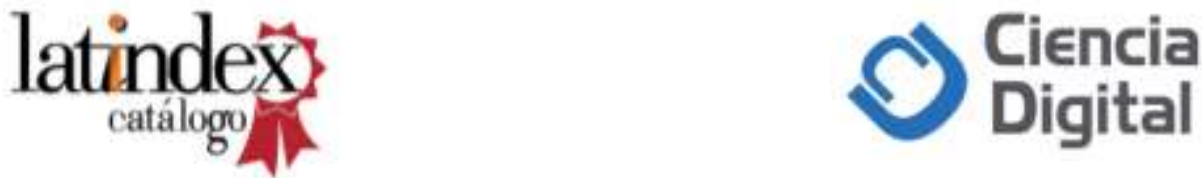